\title{
Legitimation durch Verfahren - Was bleibt?
}

\author{
Machura, Stefan
}

\section{Soziale Systeme}

\author{
DOI: \\ https://doi.org/10.1515/sosys-2017-0010 \\ Published: 06/06/2020 \\ Peer reviewed version \\ Cyswllt i'r cyhoeddiad / Link to publication
}

Dyfyniad o'r fersiwn a gyhoeddwyd / Citation for published version (APA):

Machura, S. (2020). Legitimation durch Verfahren - Was bleibt? Soziale Systeme, 22(1-2), 331354. https://doi.org/10.1515/sosys-2017-0010

\footnotetext{
Hawliau Cyffredinol / General rights

Copyright and moral rights for the publications made accessible in the public portal are retained by the authors and/or other copyright owners and it is a condition of accessing publications that users recognise and abide by the legal requirements associated with these rights.

- Users may download and print one copy of any publication from the public portal for the purpose of private study or research.

- You may not further distribute the material or use it for any profit-making activity or commercial gain

- You may freely distribute the URL identifying the publication in the public portal ?
}

Take down policy

If you believe that this document breaches copyright please contact us providing details, and we will remove access to the work immediately and investigate your claim. 


\section{Bestätigung der Autoren-Metadaten/ Author Metadata Approval Sheet}

Sehr geehrte Autoren,

Bitte prüfen Sie die unten aufgeführten Autoren-Metadaten sorgfältig und ergänzen bzw. korrigieren Sie diese ggf. in der beschreibbaren rechten Spalte.

Vielen Dank für Ihre Mitarbeit, De Gruyter

Dear author,

Please check and complete carefully the author metadata listed below by using the editable fields in the right column.

Journal-Name: Soziale Systeme

Article-DOI: https://doi.org/10.1515/sosys-2017-0010

Article-Title: Legitimation durch Verfahren - Was bleibt?

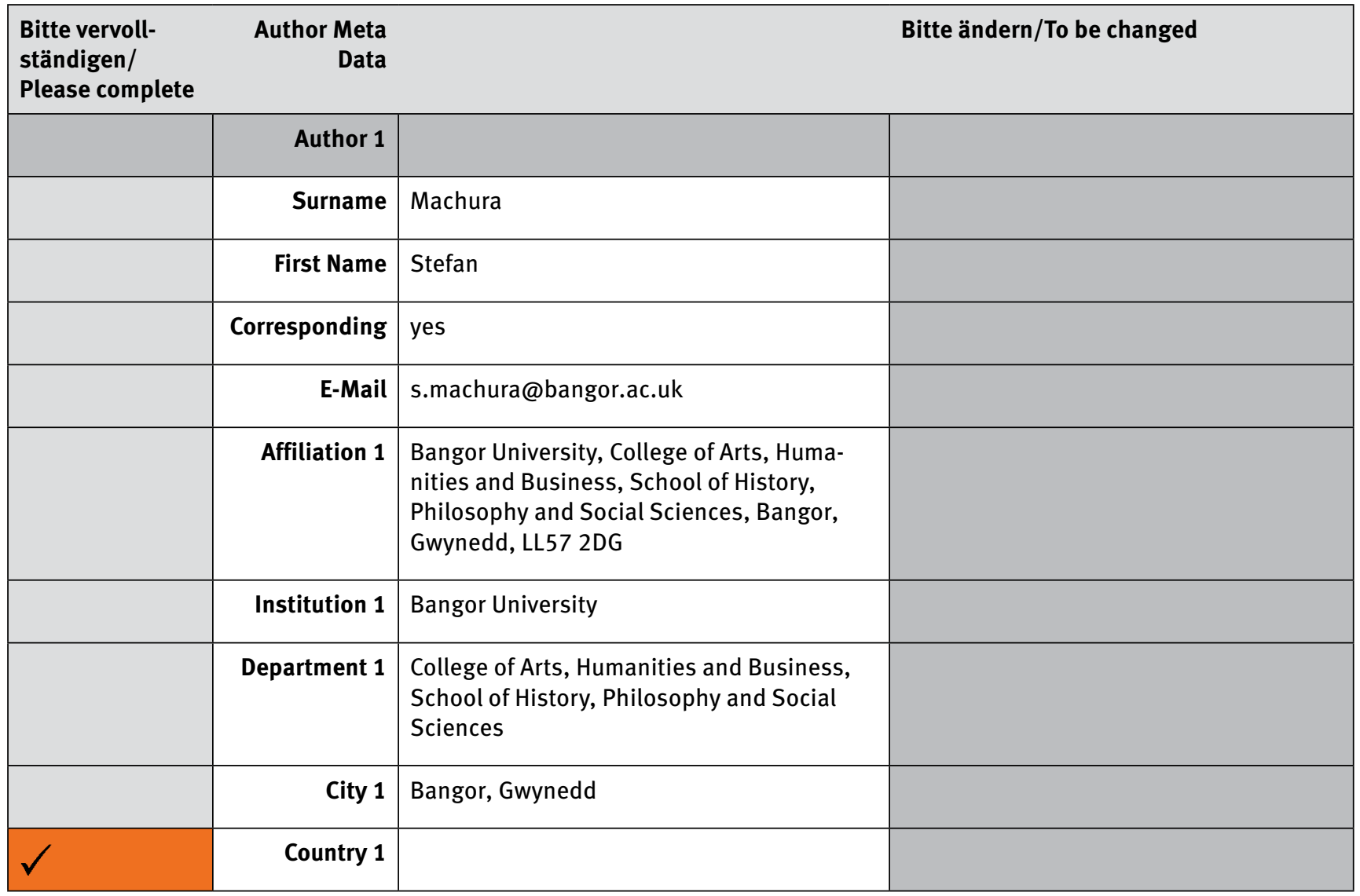




\section{Stefan Machura*}

\section{Legitimation durch Verfahren - Was bleibt?}

https://doi.org/10.1515/sosys-2017-0010

Zusammenfassung: 1969 veröffentlichte Niklas Luhmann sein Buch „Legitimation durch Verfahren“. An die Publikation schloss sich eine lebhafte Diskussion in der deutschsprachigen Literatur an. Im Gegensatz zur vorherrschenden Meinung beschrieb Luhmann, wie Institutionen ihre Legitimität selbst produzieren: Sie tun dies mittels des sozialen Mechanismus‘ des Verfahrens, wobei die individuellen Intentionen der beteiligten Parteien kaum eine Rolle spielen. Heute zeigen empirische Studien, dass die Fairness des Verfahrens entscheidend ist für die Akzeptanz der Entscheidungen und die Legitimität der Autoritäten. Darüber hinaus ist das Verhalten der Personen, die die Autorität repräsentieren, von größter Bedeutung. Der Beitrag weist nach, dass die Verfahren selbst heute fair erscheinen müssen, um Legitimation zu erlangen. Diese Entwicklung kann als eine Entwicklung von der Legitimation durch Verfahren zu Legitimation durch faire Verfahren charakterisiert werden.

Abstract: In 1969, Niklas Luhmann published his book "Legitimation durch Verfahren" (Legitimation by Procedure), which was widely quoted in German-speaking countries. This book soon provoked a fierce debate. Contrary to the established view, Luhmann stated that institutions produce their own legitimacy with the social mechanism of procedures, regardless of the intentions of the parties. Nowadays, empirical research has shown that procedural fairness is pivotal for the acceptance of decisions and for the legitimacy of the authorities. Even more, the behaviour of the people representing the authority is of paramount importance. The article argues that to elicit legitimation, procedures must demonstrate that they are fairly conducted. The development can be characterized as from legitimation by procedure to legitimation by fair procedure.

\footnotetext{
*Korrespondenzautor: Professor Dr. Dr. habil. Stefan Machura, Bangor University, College of Arts, Humanities and Business, School of History, Philosophy and Social Sciences, Bangor, Gwynedd, LL57 2DG, email: s.machura@bangor.ac.uk
} 


\section{Einleitung}

Niklas Luhmanns Werk „Legitimation durch Verfahren“ erschien $1969^{1}$ und hat gleich nach Erscheinen eine kontrovers, teilweise sogar leidenschaftlich ausgetragene Debatte ausgelöst (Machura 1993). Hochkomplexe, sich stetig wandelnde moderne Gesellschaften erforderten, so Luhmann (29f.), einen Grundkonsens, dass staatliche Entscheidungen im Regelfall hingenommen werden, sonst wären sie nicht funktionsfähig. Verfahren, die jeweils auf eine Entscheidung zuführen, sind zentral für Gesellschaften mit positiviertem Recht (232). Hagen (1972, 486) sah in Luhmanns Buch eine Theorie „des rechtlichen Positivismus und dessen Hauptproblem wiederum ist (...) seine Legitimation.“

Im politischen System selbst müsse, so Luhmann (30), Legitimität erarbeitet werden, da sie nicht mehr, etwa als Konsequenz einer von allen geteilten Weltsicht, einfach vorausgesetzt werden könne. „Legitimation“ definiert Luhmann als die „Übernahme bindender Entscheidungen in die eigene Entscheidungsstruktur" (VIIf.). Es ging ihm um eine Beschreibung von Verfahren als sozialem Mechanismus, um solche Entscheidungen herbeiführen zu können, und zwar unabhängig von der „,sachlichen Richtigkeit von Argumenten“ (5), über die Meinungen weit auseinandergehen können. Es ginge darum, inhaltliche Sicherheit für Verhalten durch „Differenzierung und Wiederverknüpfung von Verfahren“ zu ersetzen (2). Bei Legitimation durch Verfahren handele es sich um einen Lernprozess, der auf eine Umstrukturierung von Erwartungen abziele. Verlierer lernen, dass sie ihre Sicht nicht werden durchsetzen können. Funktion des Verfahrens sei nicht nur die Entscheidung, sondern auch „die Spezifizierung der Unzufriedenheit und die Zersplitterung und Absorption von Protesten“ (116; auch Luhmann 1999 b, 133). Das Umlernen wird durch einen Kommunikationsprozess erreicht, der nach rechtlichen Regeln abläuft (37; zum Kommunikationsbegriff Luhmann 1995 b, 115). In diesem Sinne könne man von einer Selbstlegitimation rechtlich verfasster Institutionen, eben durch Verfahren, sprechen.

Kritiker vermissten das aus deren Sicht Entscheidende: Die inhaltliche Qualität der Entscheidungen allein könne Legitimität begründen. In der Rechtswissenschaft dominierte eine Sicht, die Verfahren in einer „dienenden Funktion“ sieht: Das Verfahren sei Instrument zur Erlangung des „richtigen“, „gerechten“ Urteils

\footnotetext{
1 Der Erstauflage von 1969 folgte 1975 eine zweite, um ein neues Vorwort erweiterte Auflage (beide im Luchterhand-Verlag). In diesem ergänzenden Vorwort reagierte Luhmann auf seine Kritiker. Nach einer dritten, unveränderten Auflage 1978 erscheinen alle weiteren Ausgaben seit 1983 im Suhrkamp Verlag Im Folgenden wird das Buch nach der Suhrkamp-Ausgabe von 1983 zitiert, die seiten- und textidentisch mit der dritten Auflage von 1978 ist. Alle Seitenangaben ohne Autoren- und Jahresangabe in diesem Aufsatz beziehen sich auf diesen Band.
} 
(Röhl 1994, 58), ein „Mittel zum Zweck“, wie es Luhmann (227) formuliert. Viele der damaligen Kritiker hatten für soziologische Perspektiven, wie sie Luhmann entwickelte, wenig übrig. Teils waren sie auch enttäuscht, weil das Verfahrensrecht „zu kurz“ komme (z. B. Puttfarken 1977, 496).

Bis heute, so die These dieses Beitrags, bietet Luhmann in „Legitimation durch Verfahren“ wichtige Einsichten in den sozialen Mechanismus von Verfahren, wie er in Gerichten, Mediationen, und weiteren Entscheidungssituationen abläuft. Der folgende Text konzentriert sich vor allem auf Anstöße, die von Luhmanns Modell des Gerichtsverfahrens als legitimierendem sozialen Handlungssystem ausgegangen sind. Auch in späteren Veröffentlichungen hat Luhmann an dem Kerngedanken von "Legitimation durch Verfahren” in Bezug auf Gerichtsverfahren festgehalten (Kieserling 2010, 110; insbesondere Luhmann 1995 a, 208f.). Die an „Legitimation durch Verfahren“ anschließende Debatte hat sich ohnehin auf Luhmanns Modell des Gerichtsverfahrens konzentriert. Aber auch außerhalb der Gerichte finden sich ähnliche Handlungssysteme, die rechtlich vorstrukturiert sind, Beteiligten einen schrittweisen Kommunikationsprozess auferlegen und in eine Entscheidung münden.

Legitimation durch Verfahren ist dabei nicht auf die Sphäre staatlicher Strukturen beschränkt, auch große Nichtregierungsorganisationen, Kirchen, Unternehmen halten für die Konfliktbearbeitung Verfahren vor, die nach Vorschriften ablaufen. Allerdings wird man je nach Fall von den Annahmen in Luhmanns Modell des Gerichtsverfahrens Abstriche machen müssen. Ein Mediator z. B. urteilt nicht, oder wenn sich ein Beschäftigter gegen eine vom Vorgesetzten ausgesprochene Disziplinarmaßnahme in einem betriebsinternen Beschwerdeverfahren wehrt, kann es an der Unabhängigkeit der Entscheider fehlen.

Auch Gerichte kennen andere Verfahrensweisen als nur das von Luhmann behandelte, mit einem Richterspruch endende, streitige Verfahren. ${ }^{2}$ Manche Verfahren brechen mit einer Einigung ab. So können Strafverfahren in einen „Deal“ zwischen Staatsanwaltschaft und Angeklagtem münden. ${ }^{3}$ Zivilgerichte sind teilweise gehalten, einen Einigungsversuch zu unternehmen (,obligatorische Streitschlichtung“), bevor es zu einer Hauptverhandlung kommt. In Nachbarschaftsstreitigkeiten sehen einige Bundesländer den Gang zu einer Schiedsperson oder Gütestelle vor. Manche Richter machen den Parteien auch noch im Hauptverfahren Einigungsvorschläge, die von den Kontrahenten ernst genommen werden müssen, da möglicherweise ein ähnliches, oder noch ungünstigeres Urteil ergehen könnte. Alle diese Konstellationen sind für eine umfassendere

2 Siehe auch die Beiträge von Heck (2017) und Jost (2017) in diesem Heft.

3 Dazu der Beitrag von Starystach (2017) in diesem Heft. 
Theorie der Gerichtsverfahren von Interesse, die über das durch Richter streitig entschiedene Gerichtsverfahren hinausgehen. Eine solche Theorie kann auch durch Elemente des Luhmannschen Verfahrensmodells informiert sein.

Unabhängig von Niklas Luhmanns Buch gab es weitere Forschungen, insbesondere empirische Tests der Eigenschaften verschiedener Typen von Gerichtsverfahren, wobei das individuelle Bewerten und Erleben im Vordergrund stand. Tom Tyler und Allan Lind betonten den Gruppenbezug der Beteiligten, die am Verhalten der Personen, die die Institutionen im Verfahren vertreten, ihren eigenen Status in der Gruppe und den Zustand der Gruppe selbst ablesen. Verfahren müssen fair ${ }^{4}$ sein, sie haben geltenden Werten und Normen zu entsprechen. Diese Perspektive ist international vielfach empirisch bestätigt worden. Die im Folgenden dargelegte Entwicklung könnte man zusammenfassen mit der Formulierung „von Legitimation durch Verfahren zur Legitimation durch faire Verfahren“.

\section{Ausgangslage}

Max Weber (1980, 19f., 124-127) beschrieb in seiner Herrschaftssoziologie legalrational begründete Legitimität: Soziale Ordnungen werden befolgt, $u$. a. wenn sie in anerkannten Verfahren gesetzt wurden, und Befehle werden eingehalten, wenn Autoritäten durch Recht befugt sind. Wie genau Legitimation durch Verfahren funktioniert, bleibt bei Weber offen. Diese theoretische Leerstelle zu füllen, war ein Anliegen Niklas Luhmanns (31), als er sein Verfahrensbuch schrieb. Eingeflossen sind persönliche Einsichten Luhmanns (deutlich auf 93, Fn. 3). Verfahren konnte er in einer Vielzahl von Funktionen beobachten: als Rechtsreferendar, Mitarbeiter einer Anwaltskanzlei, dann auch ,juristischer Hilfsarbeiter“ am Oberverwaltungsgericht Lüneburg, später als mit Wiedergutmachungen beschäftigter Ministerialbeamter und schließlich als Landtagsreferent im niedersächsischen Kultusministerium (Treibel 1993, 20 f.; Luhmann/Hagen 2005, 20-25; Bahners 2018). In rechtlichen Streitigkeiten ist $\mathrm{zu}$ sehen, wie die Parteien vor Gericht ziehen und sich in eine Auseinandersetzung verstricken, an deren Ende ein für viele von ihnen nachteiliges und oft überraschendes Ergebnis steht. Die Ministerialbürokratie nimmt eine Schlüsselstellung im politisch-administrativen System ein (Machura 2013), in dem Verfahrensordnungen die Interessenverfolgung der Akteure strukturieren, die sich auf das politische Parkett wagen.

4 „Fairness“ und „Verfahrensgerechtigkeit“ beziehen sich auf die Art und Weise, wie ein Verfahren abläuft und werden so in diesem Beitrag verwandt. 
Luhmann (199) stellt Verfahren vor als „die Form, in der das politische System zu seiner eigenen Legitimität beiträgt“. Es geht also um die dem politischen System typische Art und Weise, wie es zusätzlich zu anderen Quellen, aus denen ihm Legitimität zufließt, selbst Legitimität (mit-)herstellen kann. Luhmann wäre also missverstanden, wenn er als jemand angesehen würde, der die inhaltliche Korrektheit oder auch nur weithin gefühlte Nützlichkeit von Entscheidungen als Legitimitätsquelle leugnet. Ausgeschlossen ist auch nicht, dass Bürger dem Staat aus inhaltlicher Überzeugung von der Vorbildlichkeit seiner Ordnung (Weber 1980, 16) Vertrauen gegenüber bringen und daher gefällte Entscheidungen regelhaft akzeptieren. Es geht darum, Legitimation durch Verfahren soziologisch zu begreifen. Auch eine Handlungsanweisung für Akteure, etwa Richter oder Ministerinnen, war von Luhmann nicht beabsichtigt.

\section{Das Verfahren als Handlungssystem}

Verfahren sind nach Luhmann eigene Handlungssysteme, mit einem die Teilnehmenden verbindenden Sinn. Verfahren führen schrittweise von einer offenen Ausgangssituation zu einer Entscheidung. ${ }^{5}$ Nach Luhmann findet sich zunächst Unsicherheit, ob man seine Rechte gegenüber anderen wird durchsetzen können, oder ob man genug politische Überzeugungskraft wird aufbringen können. In dieser Lage sind die Akteure gezwungen, Selektionen vorzunehmen: Werden sie Klage erheben und welche Argumente werden sie anführen, oder wen können sie für ihre Sache gewinnen und auf welche Weise? Für die Akteure haben Verfahren gemeinsame Bedeutungen, einen identifizierbaren sozialen Sinn (VII, 42f.). Es entwickelt sich eine Verfahrensgeschichte, die die Akteure bindet (2017, 40, 43-46). Die für die Interessenverfolgung angebotenen Verfahren bieten die Chance, sich durchzusetzen, jedoch sind die Handelnden gezwungen, in ihnen soziale Rollen aufzunehmen (47). Hier macht Luhmann also eine Anleihe an der zu dieser Zeit bereits entwickelten Rollentheorie. Rollen sind immer mit einem sozialen Status verbunden und setzen ihre Träger sozialen Erwartungen aus. Genauso verhält es sich in Verfahren, die den Klägern oder den politischen

5 Ein Eingangszeremoniell, wie bei Gericht (92), mag den Parteien das Einfinden erleichtern. Doch gibt das Zeremoniell keinesfalls die Richtung, die das Verfahren nehmen wird, vor, außer, dass es markiert, dass es zu einer bindenden Entscheidung kommen wird. Die Entscheidungsfähigkeit wird auf Seiten des Rechts durch konditionale Programmierung nach einem Wenndann-Schema erleichtert (129-133). Der Fiktion nach sind alle Konfliktkonstellationen bereits im Recht vorbedacht, der Richter wird davon entlastet, die anzuwendenden Gesichtspunkte allzu aufwendig finden zu müssen. 
Akteuren Handlungsweisen vorschreiben. Weiterhin werden Verfahrensrollen von anderen sozialen Rollen der Teilnehmer getrennt, was die Autonomie der Verfahren stärkt (48f., 64f., 120). Dazu kommt, dass Verfahren rechtlich gerahmt sind (42f.). Nach Luhmanns Definition geht es bei Recht um stabile ErwartungsErwartungen. „Das Recht prozessuiert auf eine Weise, die kein anderes System leistet, normative Erwartungen, die mit Durchhaltefähigkeit ausgestattet sind“ (Luhmann 1999 c, 4). Wer sich an einem Gerichtsverfahren beteiligt, weiß, wie auch immer ungenau, dass die anderen Personen hier, etwa Richter oder Anwälte, oder das Publikum, auf bestimmten Verhaltensmustern beharren werden. Man kann auch sagen, welche Themen zu einem Verfahren gehören und welche nicht. Damit kann man sich auf den Verhandlungsgegenstand konzentrieren. An anderer Stelle sprach Luhmann $(1981,71)$ von „spezialisierte[n] Interaktionssystemen“, die „eine Kombination von spezifischer Sensibilität und Indifferenz im übrigen“ erreichen, ,und dadurch Motivlagen generalisieren und auf das Niveau erforderlicher Entscheidungsfreiheiten" bringen.

Die verlierende Partei, so Luhmann in „Legitimation durch Verfahren“, lernt durch Enttäuschung ihrer Erwartungen und muss - manchmal zähneknirschend - das Ergebnis akzeptieren (33 f., 111 f., 236 f.). Davon unabhängig gehört zur Rolle der Parteien, dass sie sich im Prozess als lernfähig darstellen, besonders gegenüber dem Richter, auch wenn dem nicht so ist (Kieserling 2012, 146). Dies wird deutlich bei der Arbeit der Anwälte, die um dieses Erfordernis wissen und gegebenenfalls sogar anstelle begriffstutziger Klienten andeuten, dass diese die Rechtsauffassung des Gerichts teilen (werden). ${ }^{6}$ Luhmann $(33,111)$ betrachtet nur am Rande den Fall, dass die Verlierer einsichtig geworden sind und nun selbst davon überzeugt sind, dass ihre Annahmen falsch waren. Luhmann rührt an einen der Kerngehalte des Legitimitätsbegriffs bei Max Weber (1980): Die legitime Herrschaft oder die legitime Ordnung muten Menschen zu, gegebenenfalls ihrem Interesse entgegen zu handeln, auch wenn es ihnen zutiefst zuwider sein sollte. Speziell für soziale Situationen dieser Art wird der Legitimitätsbegriff vorgehalten. An dieser Stelle leistet er mehr als der Begriff des „Vertrauens“, des Vertrauens etwa in die staatlichen Institutionen. „Legitimation durch Verfahren“ führt dazu, dass Nachteiliges akzeptiert wird.

In seiner Analyse zeigt Luhmann die Mechanismen auf, mit denen Legitimation durch Verfahren arbeitet. Nur wer die Regeln anerkennt, kann teilnehmen (195). Die Parteien sind gezwungen, Rollen im Verfahren aufzunehmen. Sie

6 In einer Fußnote reißt Luhmann (98, Fn. 14) die in der Rechtspraxis so wichtige Möglichkeit des Umlernens vor dem Verfahren an. Das gehört zur Ratgeberrolle der Anwälte. Im Straf- und Jugendstrafprozeß kommen noch die Interventionen der Bewährungshelfer, Sozialarbeiter und Heimbetreuer dazu, die Angeklagte schon vor dem Prozeß bearbeiten. 
müssen dann ihre Position konsistent formulieren und sind unter Zugzwang, auf die Argumente der Gegenseite zu reagieren. Dadurch schließen sie Möglichkeiten aus, die ursprünglich im Raum standen, und enden in einer Lage, in der nur noch wenige Alternativen übrig sind. Nun, wenn „,brauchbare Entscheidungsgesichtspunkte“ herausgefiltert sind (4), kann der Richter entscheiden. Luhmann (115) spricht vom „Trichter des Verfahrens“(siehe Abbildung 1). Da sie „freiwillig“ teilgenommen haben, müssen die Parteien das Ergebnis akzeptieren. Eine politische Partei etwa, die zu Wahlen angetreten ist, gegen Konkurrenten gekämpft, und Debatten geführt hat, muss sich dem Wahlergebnis unterwerfen. Verlierer müssen feststellen, dass sie sich in einem Kommunikationsprozess selbst sozial isoliert haben (117 f.). Luhmann nennt es „expressive Isolierung der Betroffenen“ (217). ${ }^{7}$ Was in der politischen Arena etwa ein Anstoß zur breiten Mobilisierung von Mieterinteressen hätte sein können, wird vor der Zivilrichterin zu einem alltäglichen Streit zwischen Mieter und Vermieter um ein präzise umrissenes persönliches Problem. Der unterlegene Kläger kann seine Sache nicht mehr als eine Allgemeine darstellen, es war sein ganz persönlicher Konflikt, der entschieden wurde.

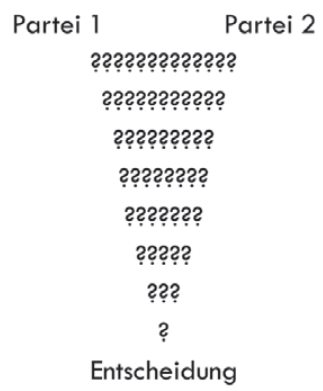

Abbildung 1: Der „Trichter des Verfahrens“

Quelle: in Anlehnung an eine Graphik von Monika Lang (1993/94).

7 Schaper (1985, 235f.) wendet ein, dass die Vereinzelung der Parteien bereits vor Beginn eines Gerichtsverfahrens beginnt, seien diese doch kaum auf die ,angemessene Verarbeitung breit solidarisierter Interessen“ eingerichtet. Luhmann (122) selbst spricht davon, dass nur diejenigen zu einer Rolle im Gerichtsverfahren zugelassen werden, die ein ganz spezifisches rechtliches Interesse vorweisen könnnen. Ein anderer Aspekt ist, dass (Gerichts-)verfahren zur Bearbeitung komplexer Problemstellungen erst nach Präparierung und Vereinfachung geeignet sind (231). Das ist eine wichtige Funktion der prozessvorbereitenden Juristen. 
Luhmann trennt konzeptionell Entscheidung und Verfahren. „Verfahren werden als soziale Systeme gesehen, die mit Entscheidungsprozessen synchronisiert, aber nicht identisch sind.“ (3) Das Verfahren als sozialer Mechanismus ermöglicht, dass entschieden werden kann, die Entscheidung selbst kann auch auf ganz anderen Faktoren beruhen. „Selbst die Prozeßordnungen der Gerichte regeln nicht den selektiven Prozeß des Erzeugens und Ausscheidens anderer Möglichkeiten, nicht die eigentliche Herstellung der Entscheidung, sondern allenfalls die Darstellung der Herstellung der Entscheidung“ (175). Auch anderswo in der rechtssoziologischen Literatur wird diese Differenz zwischen Darstellung und Herstellung richterlichen Entscheidens hervorgehoben. ${ }^{8}$

„Soziologisch gesehen schließt ein Verfahren nicht nur mit einer einzigen, rechtlich bindenden Entscheidung ab“ (3). Luhmann (3f.) nennt als zusätzliche Ergebnisse Prämissen für das weitere Verhalten der Beteiligten, das Umlernen der Parteien, Konsequenzen für ihre Stärke und Motivation, sowie die „Konfliktdämpfung“.

\section{Die Hintergrundannahmen „generalisiertes Systemvertrauen" und Offenheit}

Luhmann setzt in „Legitimation durch Verfahren“ eine Gesellschaft gewissermaßen im Normalzustand voraus, in der „generalisiertes Systemvertrauen“ besteht (193). Oder in der weitreichender Konsens zur Anerkennung von Entscheidungen doch wenigstens „durch Nichtäußerung von Dissens fingiert“ werde (122f.). Das „Ausmaß der institutionellen Anerkennung der Gerichtsbarkeit schlechthin“ sei für Legitimation durch Verfahren wichtig (17). ${ }^{9}$ Legitimität ist „eine generalisierte Bereitschaft, inhaltlich noch unbestimmte Entscheidungen innerhalb gewisser Toleranzgrenzen hinzunehmen“ (28). Legitimation durch Verfahren funktioniert, weil die Gesellschaft von den Verlierern erwartet, dass sie sich fügen (34). Die in Wahlen unterlegene Partei soll nicht zu den Waffen greifen können, wer ein Gerichtsverfahren rechtskräftig verloren hat, wird andere nicht zum gemeinsamen Widerstand gegen die Gerichtsvollzieher motivieren können.

\footnotetext{
8 Das Gemeinte wird besonders deutlich in Rüdiger Lautmanns auf teilnehmender Beobachtung als Richter beruhender Studie „Justiz - Die stille Gewalt“ (1972).

9 Jedoch konstatiert Luhmann (112) auch die „Verbreitung eines negativ stereotypisierten Bildes des Juristen und des Richters in der Öffentlichkeit“. Es diene Verlierern als „ein noch relativ unschädliches Ventil“. Empirisch aber ist das Vertrauen in die Gerichte in Deutschland hoch, wenn auch die Öffentlichkeit nicht völlig unkritisch ist (Köcher 2014, Tabelle A1).
} 
Nur in extremen Fällen kann sich, z. B. ein Verdächtiger vor gerichtlichen Anordungen drücken, indem er in die Botschaft eines Landes mit sympathisierender Regierung flüchtet. Immer häufiger formieren sich Interessengruppen, die mit Spendenaufrufen an die Öffentlichkeit langjährige politische Kampagnen und Musterprozesse beginnen, aber bis sie Erfolg haben, wird das Verfahrensergebnis stehen.

Darüber hinaus müssen in den Verfahren selbst gewisse Voraussetzungen vorliegen. Luhmann erwähnt, dass Verfahren nicht von vornherein auf ein bestimmtes Ergebnis abgestellt sein dürfen. Dann wären sie bloße Rituale (40). Auch müssen Richter Unabhängigkeit darstellen (116). Nur die Ungewißheit über den Ausgang, die immer noch bestehende Chance, motiviert die Beteiligten, sich auf ein Verfahren einzulassen, an ihm zu partizipieren und am Ende das Ergebnis anzuerkennen (51). Wenn Verfahren auf genau ein Ergebnis zulaufen, ohne dass andere denkbar wären, dann funktioniert ihr sozialer Mechanismus nicht. Ohne die Unabhängigkeit der Richter werden Prozesse fragwürdig, denn die Ungewißheit ist der „Motor des Verfahrens“ (116). Doch an dieser Stelle ist die Entwicklung nicht stehengeblieben, mehr wird von Verfahren verlangt. Bezeichnend ist, dass sich in der Bundesrepublik zunehmend die Freiheit auch der Staatsanwälte von politischen Weisungen durchsetzt.

\section{Von „Legitimation durch Verfahren“...}

Luhmanns Theorie des Verfahrens hat Anhänger gefunden, aber auch entschiedene Abwehr. Von Seiten der Kritiker wurde Luhmann im Kern vorgehalten, dass er Entscheidungsinhalte vernachlässige. Es komme auf die inhaltliche Motivation zur Anerkennung von Herrschaft und von Entscheidungen an (Machura 1993, 101-105 m.w.N). Etwas so Technisches wie Verfahren könne - gemeint war eigentlich: sollte - niemals eigene legitimatorische Kraft entfalten. Rückblickend äußerte Luhmann (1995 b, 332), seine Kritiker hätten zu viel in das Buch hineingelesen. So ginge es „nicht darum, ob und wie mit Hilfe von Verfahrensbedingungen die ,Wahrheitsfindung“ (...) oder die Anerkennung vernünftiger Geltungsansprüche erreicht werden sollte“, stattdessen gehe es um Rahmenbedingungen, wie Streit zu einem Ende gebracht werden kann.

Exemplarisch seien hier drei Kritiken aus jeweils unterschiedlicher Richtung angeführt. Luhmanns Buch enthielt für Wilhelm Hennis (1976, 19) „eine Theorie der Legitimität als Anweisung, wie man das Problem durch eifriges Kehren, sprich Verfahren, aus dem Wege schafft“. Jürgen Habermas (1971, 244) insistierte: „Ein Verfahren kann stets nur indirekt, durch Verweisung auf Instanzen, 
die ihrerseits anerkannt sein müssen, legitimieren. “ Letzlich hielt er, wie auch andere, die „Anerkennungswürdigkeit“ des politischen System für entscheidend (Habermas 1976, 39, 46, 58). Hermann Klenner (1976, 17) verstand Luhmann so, dass die soziale Funktion von Gerichtsverfahren darin bestehe, „Vertrauen zu systemkonformen Entscheidungen zu produzieren und die reibungslose Funktionalität des Rechts permanent zu gewährleisten.“ Es gehe um „das Interesse des Kapitalisten (...), in das Gewissen des Arbeiters einzudringen und dort einen inneren Aufseher in Gestalt moralischer Prinzipien des Unternehmerstandpunktes einzusetzen“. In dieser Diskussion schillern empirische und wertende Aspekte vieldeutig. Was aber, wenn die Empirie die Verfahrenstheorie Niklas Luhmanns bestätigt und noch darüber hinausweist? Seit den 1970 er Jahren hat sich eine breite Forschung entwickelt zu den Eigenschaften und Konsequenzen fairer Verfahren.

\section{... zu „Legitimation durch faire Verfahren“}

Faire oder unfaire Verfahren haben einen Effekt auf die Akzeptanz der Entscheidungen und die Legitimität der Autoritäten und der Institutionen, die das Verfahren veranstalten und führen. Damit sind noch nicht einmal alle Weiterungen benannt, die in den folgenden Jahrzehnten aufgezeigt worden sind.

Niklas Luhmanns Verfahrensbuch ist nie ins Englische übersetzt worden. ${ }^{10}$ Daher ist „Legitimation durch Verfahren“ im internationalen, insbesondere anglo-amerikanischen Raum, nahezu unbekannt. Es hat aber eine weit ausgreifende empirische Forschung zu den Eigenschaften und Konsequenzen als fair oder unfair wahrgenommener Verfahren gegeben. Sie war zunächst von den Experimenten, die Thibaut und Walker in ihrem Buch „Procedural Justice“ (1975) zusammengefasst haben, inspiriert ${ }^{11}$. Thibaut und Walker knüpften an die sozialpsychologische Equity Theory (Walster et al. 1973) an. In ihrem Mittelpunkt steht, wie am Eigennutzen orientierte Akteure - die auch Luhmann (2) voraussetzt - Verteilungsentscheidungen bewerten. Thibaut und Walkers Idee ist nun, dass ein faires Verfahren den Beteiligten erlaubt, ihre Sicht umfassend darzulegen, so dass eine gerechte Verteilungsentscheidung gefällt werden kann. Ent-

10 Ein knapper Anriss des Inhalts findet sich im Kapitel „Legitimität“ in der „Rechtssoziologie“, die in englischer Sprache verfügbar ist (Luhmann 1983 b; 1985).

11 Im internationalen Maßstab eher unbeachtet blieben die ebenfalls experimentellen Studien von Tausch (Tausch/Langer 1971; Tausch et al. 1975), denen es aber auch an der Zuspitzung fehlte, wie sie für Thibaut und Walker charakteristisch ist. 
scheidend ist also process control der Parteien - so wie der amerikanische Richter den Parteien und ihren Anwälten die Beweiserhebung überlässt. Häufiger wird auch von voice gesprochen. Das entspricht teilweise dem, wie Luhmann Verfahrensabläufe vor Gericht beschreibt ${ }^{12}$, allerdings fehlt das bei Luhmann betonte Element der unfreiwilligen Verstrickung der Beteiligten, oder gar ihrer „Schwächung und Zermürbung“ (4), und viel von der soziologischen Rahmung, alles Elemente, die über das sozialpsychologische Axiom der nach Eigennutz strebenden Akteure hinausweisen.

Zunächst stellten an Thibaut und Walker anschließende Studien fest, dass Probanden Verfahren auch dann als fair bewerten, wenn sie verlieren, so lange sie nur Gelegenheit hatten, ihre Meinungen auszudrücken (Lind/Tyler 1988). Thibauts und Walkers Idee, dass der Wert eines Verfahrens letztlich darin liegt, zu einem guten Ergebnis zu kommen, wurde infrage gestellt. Auch voice erschien nicht mehr als der Hauptfaktor für Verfahrensgerechtigkeit (Lind 1994 c, 4 f.). Die Ergebnisse empirischer Forschung deuteten auf einen „fair process effect“ hin: Negative Ergebnisse werden hingenommen, wenn das Verfahren als fair erscheint (Greenberg/Folger 1983; Leventhal 1980, 36; Hough 2013). Verfahrensgerechtigkeit kann auch unabhängig vom Ergebnis bewertet werden (Landis/ Goodstein 1986, 682; Lind et al. 1993, 226). Als fair empfundene Verfahren können dazu führen, dass Parteien, die sich nicht auf eine gerechte Entscheidung einigen können, eine Lösung akzeptieren (Röhl 1993, 21-25).

Nach Thibaut und Walker ist voice/process control für Verfahrensgerechtigkeit entscheidend. In seinem Artikel „What Should Be Done With Equity Theory?” erweiterte Leventhal (1980, 39-46) den Kreis der Kriterien fairer Verfahren. Er benennt:

- Konsistenz

- Unvoreingenommeneit

- Sorgfalt

- Repräsentanz

- Korrigierbarkeit

- Moralität.

Am Beispiel des Gerichtsverfahrens in John Fords Film „Prisoner of Shark Island“ (USA 1936) lassen sie sich verdeutlichen. Der Film beruht auf dem historischen Prozess von 1865 gegen Dr. Samuel Mudd, der wegen angeblicher Teilnahme am Mordkomplott gegen Abraham Lincoln verurteilt worden war. Dabei war sein ein-

12 Hiergegen wandte Esser ein, dass der deutsche Richter im Zivilverfahren eine stärker steuernde Rolle hat (Esser 1970, 205-209, auch Schaper 1985, 238). 
ziger Beitrag, das gebrochene Bein des ihm unbekannten Attentäters behandelt zu haben. Das Konsistenzkriterium verlangt, dass es keine Sonderverfahren gibt, die einige bevorteilen oder benachteiligen. Dr. Samuel Mudd wird als Zivilist vor ein eigens konstituiertes Militärtribunal gebracht (20th Century Fox 1969, 48). Der Prozess findet sodann in einer politisch aufgeheizten Atmosphäre vor parteiischen Richtern statt (Kriterium der Unvoreingenommenheit). Das Sorgfaltskriterium verlangt, dass ein Urteil auf einer ausreichenden Erhebung der Beweise beruht- in Fords Film hört das Gericht nur Zeugen für die Anklage. Nach dem Repräsentanzkriterium muss jede Partei ihre Sicht der Dinge darlegen können. Die Militärrichter in „Prisoner of Shark Island“ werden instruiert: „the court will ignore the remarks of the prisoner” (20th Century Fox 1969, 48). In der Justiz ist das Korrigierbarkeitskriterium durch die Möglichkeit der Berufung oder Revision institutionell gewährleistet, Mudd scheitert auch hier. Schließlich sollen nach dem Moralitätskriterium Verfahren den in einer Gesellschaft geltenden Normen und Werten entsprechen. In Fords Film betritt der Assistant Secretary of War vor Prozessbeginn den Beratungssaal der Militärrichter - alles hochrangige Offiziere - und instruiert sie, keine Gedanken an „trifling technicalities of the law“ zu verschwenden, über Beweisregeln hinwegzusehen, den Grundsatz des reasonable doubt nicht anzuwenden und aus Staatsräson abschreckende Urteile zu fällen (20th Century Fox 1969, 36-38).

Das Kriterium der Moralität bei Leventhal (1980) verdeutlicht den radikal empirischen Ansatz. „Equity is in the eye of the beholder“ (Walster et al. 1973, 152). Das ausdeutbare Kriterium kann eine Vielzahl von Anforderungen an Verfahren abdecken (Folger/Cropanzano 1998, 48). Schon bei Max Weber (1980) hing legitime Herrschaft davon ab, dass die jeweiligen gesellschaftlichen Erwartungen erfüllt werden. Zustimmend zitierte Luhmann (30) Bourricaud (1961, 7), legitim sei „un pouvoir qui accepte ou même qui institue son propre procès de légitimation“. Wenn eine Gesellschaft Maßstäbe an Verfahren anlegt, die denen der Sozialwissenschaftler widersprechen, so ist auch das ein Ergebnis der Forschung. Bierbrauer (1994, 258) z. B. fand in einer Studie, dass Befragte nahöstlicher Herkunft einen Richter dann eher als gerecht ansahen, wenn er Männern mehr Glauben schenkte als Frauen. Luhmann (30) spricht von der „Gleichheit der Chance, befriedigende Entscheidungen zu erhalten“ als Merkmal moderner Gesellschaften. Alle Ungleichheiten seien begründungsbedürftig (172). Er betonte das Erfordernis der Unparteilichkeit der Richter (1995a, 210). Als Teil der richterlichen Unparteilichkeit stellte Luhmann (134) den Aspekt der Rollentrennung heraus, an der es im Filmbeispiel fehlt, da die Richter als Offiziere auch in die Militärhierarchie eingespannt sind. Als Teil der Richterrolle sah es Luhmann, darauf hinzuwirken, dass im Verfahren allen Beteiligten übermäßige, etwa emotionale, Belastungen erspart werden, die ihnen die Teilnahme erschweren können (86f., 
106). ${ }^{13}$ Das Konsistenzkriterium taucht bei Luhmann (1999a, 390 f.) auf, wenn er sein Konzept von „Gerechtigkeit“ vorstellt: „Adäquate Komplexität des Rechtssystems“ erlaube „konsistentes Entscheiden“ im System rechtlich orientierter Kommunikationen. Ihm erschienen auch „Kontaktsysteme“ zwischen Anwälten und Richtern als riskant, wenn sich z. B. eine Partei durch die Vertrautheit zwischen diesen einen Vorteil erhofft (80f.). ${ }^{14}$

Luhmann grenzt seine Verfahrenstheorie explizit ab von einem konventionellen Verständnis von Prozessen, das ihren Zweck in der Wahrheitsfindung sieht. „Wahrheit“ ist für Luhmann (23) ganz einfach ein Mechanismus, um Unsicherheit zu reduzieren: es geht um kommunikative „Übertragung reduzierter Komplexität“. Den strengen methodischen Anforderungen an Wahrheitsermittlung, wie ihn die Naturwissenschaften geprägt haben, können die von Luhmann diskutierten Verfahrenstypen nicht gerecht werden (20). Sie wären schlicht überfordert. Ein Gerichtsverfahren erzeugt nur eine forensische Wahrheit. ${ }^{15}$ Zwar hat z. B. im Strafprozeß nach $\S 244$ Abs. 2 StPO das Gericht zur „Erforschung der Wahrheit die Beweisaufnahme von Amts wegen auf alle Tatsachen und Beweismittel zu erstrecken, die für die Entscheidung von Bedeutung sind“. In der Praxis aber geht es weniger um „die Feststellung der Wahrheit, als (...) die Ermittlung eines Sachverhalts, der das Urteil rechtfertigt und allgemein akzeptiert wird“ (Voigt 1998, 131). Ungeachtet der Skepsis unter Experten kann das von Leventhal (1980) formulierte Sorgfaltskriterium für die Bewertung der Fairness von Verfahren und der Entscheidungsträger durch Beteiligte und Dritte ${ }^{16}$ eine Rolle spielen. Entsprechend fielen auch empirische Studien mit Angeklagten, Antragsgegnern beim Schiedsamt, Klägern und Laienrichtern aus, wobei auch allgemeiner Leventhals Kriterien für Fairness empirische Bestätigung fanden (z. B. Tyler 1984; Tyler 1990; Machura 2001, 104-107, 218-221; Machura/Donskow/Litvinova 2003, 77-79; Röhl/ Weiß 2005, $248 \mathrm{f}$., auch mit Ergebnissen zu Anwälten: 255, 258, 260; Machura 2006, 78-81; Grootelaar/van den Bos 2018, 247).

In den folgenden Jahren gab es eine international und interkulturell ausgreifende Gerechtigkeitsforschung. Es stellten sich starke Divergenzen bei den Prinzi-

13 Verfahren erfordern von den Beteiligten taktvolles Vorgehen, was Luhmann (104, Fn. 10; dazu den Beitrag von Liebler/Zifonun (2017) in diesem Heft) in dem angelsächsischen Verständnis von fair trial und dem deutschen der Menschenwürde gespiegelt sieht. Gegebenenfalls werde auch auf Herausstellung der Wahrheit verzichtet.

$14 \mathrm{Zu}$ Kontaktsystemen siehe auch den Beitrag von Starystach (2017) in diesem Heft.

15 Vgl. dazu auch der Beitrag von Jost (2017) in diesem Heft.

16 Luhmann (123) erwähnt, dass Nichtbeteiligte zu dem Eindruck gelangen müssen, dass alles rechtens zugehe, „daß in ernsthafter, aufrichtiger und angestrengter Bemühung Wahrheit und Recht ermittelt werden und daß auch sie gegebenenfalls mit Hilfe dieser Institution zu ihrem Recht kommen werden.“ 
pien der Verteilungsgerechtigkeit, hingegen aber eine sehr weitgehende Ähnlichkeit bei den Kriterien für prozedurale Fairness (so schon Tyler/Lind 1992, 140-143) heraus. Um einem Missverständnis vorzubeugen: Bewertungen der Gerechtigkeit von Entscheidungen werden also nicht ausgeblendet, es zeigt sich typischerweise, dass sie auch eine Rolle für subjektive Bewertungen spielen. Studien zum Vertrauen in die Polizei und die Justiz z.B. fanden eine Abhängigkeit von Wahrnehmungen der Effektivität, der Gerechtigkeit von Entscheidungen und der Verfahrensgerechtigkeit, wobei die letztere oft entscheidender ist (z. B. Tyler/Huo 2002; Sunshine/Tyler 2003; Tyler/Jackson 2013; Machura/Love/Dwight 2014). Luhmann (218) selbst erwähnt die Rationalität und Leistungsfähigkeit einer Verwaltung als potentiell legitimationsfördernd. Das Gerichtsverfahren werde ,als ein Drama, das richtige und gerechte Entscheidung symbolisiert“ gestaltet (124).

Daran schließt Luhmann (124-128) noch heute aktuelle Überlegungen zur Funktion der Öffentlichkeit und der Medien bei der Gerichtsbeobachtung an. Für Prozeßbeteiligte dürfe die Berichterstattung und Reaktion der Öffentlichkeit nicht nachteilig werden. Die Integrität des Gerichtsverfahrens müsse gewahrt bleiben. Wie das Beispiel des „Prisoner of Shark Island“ verdeutlicht, bilden Gerichtsverfahren ein Element zahlloser Filme, Hörspiele und Fernsehsendungen. Die weithin geteilten Kriterien für faire Verfahren tragen dazu bei, dass auch Geschichten, die in fremden Ländern mit den jeweiligen Besonderheiten ihrer Justiz spielen, international verstanden werden (Machura 2005).

\section{Gruppenidentifikation und Fairness}

Luhmann beschreibt Individuen, die ihre Interessen verfolgen und vor allem an ihrem Vorteil interessiert sind ${ }^{17}$. Sie bewerteten Autoritäten, beispielsweise einen Richter (111f.), nach dem Ergebnis ihres Verfahrens. Das muss aber nicht so sein. So beurteilten z. B. in einer Studie jugendliche Strafgefangene ihren Richter nach der wahrgenommenen Fairness ihnen gegenüber (Haller/Machura 1995), in einer anderen Studie bewerteten Kläger und Angeklagte den Richter nach der ihnen gegenüber gezeigten Fairness (Grootelaar/van den Bos 2018).

Das Gruppenwertmodell der Verfahrensgerechtigkeit, wie es Lind und Tyler (1988; Tyler 1990) formuliert haben, versteht Verfahren als Symbole für die Werte einer Gruppe (Tyler/Lind 1992, 140). Menschen schätzen es, in einer Gruppe gut aufgehoben zu sein. Sie werden in eine Gruppe sozialisiert. Zu den Werten

17 Wobei Luhmann (126, 230, Fn. 15) in seinem Verfahrensbuch nicht von „rational und richtig urteilenden" Personen ausgeht. 
einer Gruppe zählen solche für faire Verfahren. Wenn nun jemand erlebt, dass sie durch die Autoritäten schlechter behandelt wird, dann tritt eine Krise der Gruppenidentifikation ein. Die Einzelne kann sich als Außenseiter definieren, die eventuell den Autoritäten und der Gruppe nichts mehr zu schulden meint. Etwas gewöhnungsbedürftig für Soziologen ist die Gleichsetzung von Gruppe und Gesellschaft, wenn es etwa um Prozessteilnehmer und Gerichtsverfahren geht. Aber das trifft nicht den Kern des Group Value Models.

Die gegenüber dem Einzelnen auftretenden Staatsautoritäten erscheinen als "personification of the dominant society" (Lind 1994 c, 10). Nach Lind und Tyler lesen die Betroffenen von ihrer Behandlung durch den Richter ab, ob sie als vollberechtigte Mitglieder der Gesellschaft gelten können. „[B] eyond communicating a message about accesss to resources, procedures communicate messages about a person’s status within society“ (Tyler/Belliveau 1995, 308f.). ${ }^{18}$

Der Ansatz von Lind und Tyler erinnert an die Soziologie Emile Durkheims (Bora 1997, 85), und wird in der Literatur auch teilweise als „neo-Durkheimian“ (Jackson/Sunshine 2007) apostrophiert. Bei Durkheim haben die Mitglieder der Gesellschaft prinzipiell ein Interesse daran, dass die grundlegenden Normen gelten, und beobachten genau, ob Regelbrecher sanktioniert werden (Durkheim 1976). Staatliche Aktivität wird von der Öffentlichkeit beurteilt; in der modernen demokratischen Gesellschaft soll sie dem Individuum dienen, seine Rechte wahren (Durkheim 1999, 100, 119f.). Nach dem Group Value Model verfolgen Gruppenmitglieder, ob Autoritäten in Verfahren den Fairnessanforderungen entsprechen, wenn nicht, dann ist eigentlich jeder auch in Gefahr diskriminiert zu werden (Tyler 1990, 174). Daher auch verwenden Menschen eine "fairness heuristic" (Lind et al. 1993, 225 ff.; Lind 1994 b): Sie ziehen aus der beobachteten Fairness der Autoritäten Schlussfolgerungen. Wenn z. B. Mächtige Richter kaufen können, dann droht die Justiz für alle zu einer Gefahr zu werden. Einige Untersuchungen zeigen, dass Menschen Fairness nicht nur für sich wollen, sondern auch für andere (Rasinski/Tyler 1988, 17; Machura 2001; Machura/Donskow/Litvinova 2003; Machura 2006, anders Boeckmann/Tyler 1997; Platow/Reid/Andrew 1998). Damit wird zugleich der Fokus, den Luhmann in seinem Verfahrensbuch auf die Isolierung und Akzeptanz der Verlierer legt, erweitert. Fairness oder Unfairness von Verfahren betrifft auch die Kläger, die Behandlung von Zeugen, etwaige

18 An einer Stelle in „Legitimation durch Verfahren“ äußerte Luhmann (215-218) die Idee, dass die Verwaltung von legitimierenden Funktionen entlastet werden könne, nun da die Politik demokratisch und die Verwaltungsgerichtsbarkeit ausgebaut sei. Die Procedural Justice-Forschung legt nahe, dass das kaum möglich sein wird, denn jede Begegnung mit Behörden kann für den Legitimitätsglauben relevant werden. Darüberhinaus spielt Fairness auch eine Rolle für die Kooperation innerhalb von Verwaltungseinheiten und zwischen ihnen (Machura 2016). 
Zuschauer und selbst die professionellen Akteure, schließlich die Öffentlichkeit, die Prozesse über Medien verfolgt.

Status recognition als gleichberechtigtes Mitglied der Gesellschaft, dass einem die Rechte gewährt werden, die allen garantiert sind, ist neben voice, Unvoreingenommenheit, sowie dem Bemühen, dem anderen gerecht $\mathrm{zu}$ werden (benevolence) eines der vier von Lind (1994 a) und Tyler (1994) herausgearbeiteten Fairnesskriterien. An diesen Voraussetzungen kann es fehlen, etwa wo der Staat sich, wie in England und Wales, weitgehend aus der Prozesskostenhilfe zurückzieht (Welsh 2017). Die Hilflosigkeit unerfahrener Teilnehmer hat Luhmann in Legitimation durch Verfahren selbst eindringlich beschrieben (46). „Praktisch nur durch Beteiligung von Anwälten“ werde die Prozeßvoraussetzung „annähernd gleicher Qualifikation“ erreicht (103, Fn. 7). Noch schlechter steht es, wo Richter Gerichtsprozesse in eine Gaudi verwandeln und die Worte der Angeklagten in allgemeinem Gelächter untergehen, so wie es Cole $(1990,314)$ für ein Gericht in Lagos beschrieb.

Tatsächlich sind in die Verfahrensregeln, etwa von Gerichtsverfahren, aber auch z.B. von Beschwerdeverfahren in Behörden, materiale Prinzipien der Verfahrensgerechtigkeit eingelassen (Zippelius 1973, 298 f.). Artikel 6 (1) der Europäischen Menschenrechtskommission begründet ein Recht auf ein unparteiisches und unabhängiges Gericht sowie auf faire und angemessen zügige Verfahren. Artikel 3 zählt weitere Rechte angeklagter Personen auf. Materiale Prinzipien werden auch in der jurististischen Literatur deutlich. Sie kennt z.B. die Grundsätze der Respektierung der Parteien als handelnde Rechtssubjekte, die Anhörung aller Seiten, die Unabhängigkeit der Richter und die Öffentlichkeit der Gerichtsverfahren (Zippelius 1973, 298f.).

Der Ansatz von Lind und Tyler kann erklären, warum Menschen etwa ein Gerichtsverfahren gewinnen, aber vom Auftreten des Richter abgestoßen sind und warum ihr Justizvertrauen dann schwindet. Oder warum jemand einen Prozess verliert, die Richterin aber als so positiv erlebt hat, dass den Gerichten weiter vertraut wird. Die von den Repräsentanten der Gruppe ausgeübte Fairness erwies sich empirisch als entscheidend. Demnach wird vor Gericht die Fairness des Verfahrens und die Geltung der Normen fairen Verhaltens an der Rollenleistung der Richter abgelesen. Das Gleiche gilt für andere Beamte, die dem Einzelnen begegnen.

Bei Niklas Luhmann Verfahrensbuch, im Kapitel zu Gerichtsverfahren, findet sich als Voraussetzung für Legitimation durch Verfahren, dass ein allgemeines Klima herrscht, in dem Verfahrensentscheidungen respektiert werden, während er an anderer Stelle erwähnt, dass das Publikum aus Verfahren lernt und „Systemvertrauen“ faßt (199). Die empirischen Studien im Anschluß an Lind und Tyler zeigen detaillierter auf, wie und unter welchen Bedingungen sich dies voll- 
zieht, nämlich durch die Wahrnehmung von Fairness in Verfahren und besonders durch die Rollenleistung der Richter bzw. anderer verfahrensprägender, die Behörden und Organisationen repräsentierender Personen.

\section{Jenseits von „Legitimation durch Verfahren“?}

Lind und Tyler verankern Verfahrensgerechtigkeit in den gesellschaftlichen (Gruppen-)Normen und in dem Bestreben der Einzelnen, innerhalb von Gruppen Sicherheit zu finden. Daher kann ihr Ansatz auch erklären, warum der, der sich in Opposition zur Mehrheit definiert, nicht notwendig Legitimation durch Fairness zulässt. Wer sich als Gegner sieht, mag rein am Eigeninteresse orientiert sein, z. B. nur am vorteilhaften Ausgang eines Gerichtsverfahrens und keinen Gedanken etwa auf die Fairness des Richters verschwenden. So hat eine niederländische Studie ergeben, dass die meisten Insassen eines Gefängnisses die Wärter nach Kriterien erlebter Fairness beurteilen, ein harter Kern aber, der sich als Gegner der Gesellschaft sieht, nur nach den erhaltenen Vorteilen (Vermunt/Blaauw/Lind 1996, 16 f.). In einer Arbeit zum Vertrauen in die Polizei hat eine Teilgruppe sich mit dem britischen Staat wenig identifizierender Muslime kein Vertrauen in die Polizei generell gezeigt, dagegen aber sehr wohl registriert, wenn sie persönlich korrekt und fair agierenden Beamten begegnet sind (Huq/Tyler/Schulhofer 2011, 752). Damit ist der Fall beschrieben, dass eine Staatsordnung nicht unterstützt wird und Legitimation durch Fairness für die Institutionen abbricht, dennoch aber universal gültige Kriterien für Fairness zur Anwendung kommen.

Organisierte Interessengruppen bilden einen anderen Bereich, in dem Legitimation durch Verfahren unter Umständen nicht trägt (122, Fn. 3). Wie von Offe (1972, 133f.) dargelegt, wird der Mechanismus „durchschaut“ und wirkt nicht mehr. Gerichtsverfahren können als Instrumente für eine breite politische Mobilisierung verwendet und Teil weiter greifender Aktionen werden (Röhl 1977, 57-66). Protestgruppen können nach durchlaufenem Mediationsverfahren dann doch Gewalt anwenden (Epp 1997, 3). Im Extremfall werden Gerichtsverfahren von politischen Kräften genutzt, um Märtyrer zu produzieren und die Legitimität des politischen Systems anzugreifen (Grunwald 2006).

Von solchen Randfällen abgesehen, die aber sicher nicht vernachlässigt werden dürfen, besteht im Regelfall der von Luhmann beschriebene Grundkonsens, dass Verfahrensergebnisse anzuerkennen sind. Wenn dann doch die im Verfahren unterlegene Seite den aus Entscheidungen folgenden Verpflichtungen nicht nachkommt, dann liegt es häufig an Unzulänglichkeiten der Person oder der Lebensverhältnisse und nicht am Fehlen eines gesellschaftlichen Grund- 
konsenses. Die Legitimität der Institutionen, der Entscheidungsträger oder der Entscheidung ist nicht infrage gestellt, wenn Personen etwa aufgrund von Mittellosigkeit, physischer oder psychischer Kondition nicht in der Lage sind, angemessene Anordnungen zu befolgen. Das aber ist häufiger der Fall als eine reflektierte, irgendwie politische Entscheidung gegen konformes Verhalten.

Weil das Fehlen einen weit verbreiteten Erwartung, dass aus Verfahren hervorgehende Entscheidungen bindend sind, in westlichen Gesellschaften äußerst selten ist, fehlt es auch an Studien aus der Procedural Justice-Forschung zu dieser Konstellation. Auch Luhmann behandelt in „Legitimation durch Verfahren“ den Fall einer Gesellschaft, die durch diesen Konsens ausgezeichnet ist. Das schließt nicht aus, dass, etwa in „failed states“, nicht nur Verfahren fragwürdig sind, sondern dass auch in der Gesellschaft die Bereitschaft fehlt, Verfahren anzuerkennen. Immerhin könnte man aus der Darstellung Luhmanns und aus den Erkenntnissen der Procedural Justice-Forschung Lehren ziehen, wie Institutionen arbeiten müssen, um Vertrauen zu gewinnen.

\section{Schlussfolgerungen}

Luhmann handelt in „Legitimation durch Verfahren“ davon, wie eine Gesellschaft und wie auch Organisationen bindende Entscheidungen herbeiführen. Den Fall, dass Beteiligte und Nichtbeteiligte eine Entscheidung akzeptieren, weil sie von deren Inhalt überzeugt sind, hat er für nicht so erklärungsbedürftig erachtet.

Luhmann liefert eine genuin soziologische Analyse von Verfahren als Handlungssystem. Als Stichworte seien genannt: Rechtliche Rahmenvorgabe, Übernahme spezifischer Rollen im Verfahren, Bindungskraft der Verfahrensgeschichte, Darstellung von Unvoreingenommenheit der Entscheider, Lernen durch Enttäuschung, soziale Isolierung der Unterlegenen und ein gesellschaftlicher Konsens, dass Verfahrensentscheidungen bindend sind. Dieser Kern ist noch heute zentral für ein Verständnis von Verfahren, dafür wie sie Beteiligte in eine Position bringen, aus der von ihnen erwartet wird, dass sie unliebsame Ergebnisse hinnehmen müssen. Nichtbeteiligte gewinnen den Eindruck, dass die Parteien die Entscheidung kommunikativ vorbereitet haben, und damit ihre Chance hatten. Auch eine gesellschaftskritische Perspektive sollte daran interessiert sein, wie genau, durch Vorliegen welcher Voraussetzungen und in welchen Schritten als negativ bewertete Benachteiligungen in Verfahren produziert werden.

Verfahren können allerdings mehr leisten, als es Niklas Luhmann in seinem Buch „Legitimation durch Verfahren“ anspricht. Luhmann demonstriert, wie 
sich ein uneinsichtiger Verlierer sozial isoliert. Jedoch wird zu sehr auf ein eng definiertes Eigeninteresse der Akteure abgehoben und das Erleben der Verfahren durch andere Beteiligte, nicht nur den Verlierer, geriet zum größten Teil aus dem Blick. Die Group Value Theory Lind und Tylers öffnet die Perspektive auf ein gesellschaftliches Interesse am Ablauf von Verfahren und auch darauf, dass Betroffene über das verfolgte Anliegen hinaus beobachten, ob ihre Rechte, ihr Status in der Gesellschaft, respektiert werden. Gute Erfahrungen tragen zur Legitimität der Institutionen bei. Auch andere sollen den geltenden Werten für Fairness entsprechend behandelt werden, sonst wäre für alle eine Diskriminierung wahrscheinlich. Am Verhalten des Personals, das die Institutionen repräsentiert, der Richter, der Polizisten, der Lehrer oder Vorgesetzten, wird abgelesen, wie gut sie funktionieren. Damit wird die auch bei Luhmann mitbedachte Rollenleistung, etwa der Richter, stärker gemacht und auch nahegelegt, dass durch entsprechende Auswahl und Training des Personals ganz praktisch Verbesserungen möglich sind. Logischerweise gerät damit auch die Ressourcenausstattung in den Blick, die sorgfältig und fair geführte Verfahren ermöglichen muß. Ein umfassenderes Verständnis von „Legitimation durch Verfahren“ weist praktischen Lösungen für ganz konkrete Alltagsprobleme die Richtung.

Inhaltliche Anforderungen sind in Verfahrensregeln eingelassen und Rollenerwartungen für Autoritäten sind durch Fairness mitdefiniert. Legitimation durch Verfahren scheint nach allem ein sehr robuster Mechanismus zu sein und interkulturell vergleichende Studien haben gezeigt, dass weithin geteilte Anforderungen an als fair empfundene Verfahren existieren.

Luhmann sieht Legitimation durch Verfahren im Kontext einer modernen Gesellschaft, in der sich alles Recht durch Entscheidung ändern kann. In der Abfolge von Verfahren können verschiedenste Interessen befriedigt werden, etwa in Gesetzgebungsprozessen. In Gesellschaften, die politisch gespalten sind und in denen rivalisierende Gruppen darum konkurrieren, auf wessen Seite die staatlichen Institutionen stehen, kann Legitimation durch faire Verfahren möglicherweise ausgleichend wirken. So können Organisationen durch betont faire und respektvolle Behandlung von Bürgern ein Vertrauenspolster schaffen, das es ermöglicht, Forderungen einer Seite nachzukommen, ohne dass die andere sich hintergangen fühlt.

Niklas Luhmanns „Legitimation durch Verfahren“ wird durch die Procedural Justice-Forschung ergänzt: sie beschreibt näher, welche Bedingungen ein Verfahren erfüllen muss, um als fair erlebt zu werden. Das Group Value Model von Lind und Tyler gibt dazu noch das Element der Gruppenzugehörigkeit. Damit wird erklärbar, warum in manchen Konstellationen „Legitimation durch Verfahren“ nicht funktioniert. Wenn die hinter einem Verfahren stehende Ordnung einer abgelehnten sozialen Gruppe (oder Mehrheitsgesellschaft) zugeschrieben 
wird, dann kommt es nur noch auf die Günstigkeit des Verfahrensergebnisses an. Die Group Value Theory sieht Verfahren überdies als Symbole für eine Gruppe (Gesellschaft) und ihre Werte. Sich in Opposition definierende Gruppen können auch deshalb besonders dafür sensibilisiert sein, mit der Anerkennung von Verfahren und ihren Ergebnissen nicht auch die abgelehnte soziale Ordnung zu unterstützen. Für die Procedural Justice-Forschung, wie sie sich insbesondere im Anschluss an die Arbeiten der Sozialpsychologen Leventhal, Tyler und Lind entwickelt hat, bietet Luhmann ein Modell des Verfahrens als rechtlich gerahmter, schrittweiser Interaktion zwischen Rollenträgern. Darüber hinaus gibt er auch Bedingungen an, die für das Funktionieren von Legitimation durch Verfahren in Gesellschaft, Staat und Recht gegeben sein müssen, Bedingungen, die in der Procedural Justice-Forschung häufig thematisiert worden sind.

Legitimation durch Verfahren erscheint als ein essentielles Konzept für eine adäquate Gesellschaftsanalyse. Der Kerngedanke in Niklas Luhmanns Buch ist auch fünfzig Jahre nach seiner Veröffentlichung aktuell. Die neueren Erkenntnisse aus der Procedural Justice-Forschung bieten eine Ergänzung und Abrundung. Beides zusammengenommen ergibt ein Bild: Faire Verfahren sichern nicht nur die Akzeptanz von Entscheidungen, sondern stärken soziale Bindungskräfte. Ganz im Sinne Luhmanns kann man festhalten: Legitimität muss im Alltag erarbeitet werden.

\section{Literatur}

$20^{\text {th }}$ Century Fox (1969): The Prisoner of Shark Island. Continuity and Dialogue. Drehbuch.

Bahners, Patrick (2018): Danach fragt ein neuer Beamter nicht. Frankfurter Allgemeine Zeitung, 17. Januar 2018, N 3.

Bierbrauer, Günter (1994): Toward an Understanding of Legal Culture: Variations in Individualism and Collectivism Between Kurds, Lebanese, and Germans. Law and Society Review 28, 243-264.

Boeckmann, Robert J./Tyler, Tom R. (1997): Commonsense Justice and Inclusion Within the Moral Community. When Do People Receive Procedural Protections From Others? Psychology, Public Policy, and Law 3, 362-380.

Bora, Alfons (1997): Procedural Justice as a Contested Concept, in: Klaus F. Röhl/Stefan Machura (Hrsg.), Procedural Justice. Aldershot: Ashgate, 81-104.

Bourricaud, François (1961): Esquisse d'une théorie de l'autorité. Paris: Librairie Plon.

Cole, Bankole A. (1990): Rough Justice: Criminal Proceedings in Nigerian Magistrates' Courts. International Journal of the Sociology of Law 18, 299-316.

Durkheim, Emile (1976): Regeln der soziologischen Methode, hrsg. von René König. 4. Auflage. Neuwied: Luchterhand.

Durkheim, Emile (1999): Physik der Sitten und des Rechts, hrsg. von Hans-Peter Müller. Frankfurt a.M.: Suhrkamp. 
Epp, Astrid (1997): Divergierende Konzepte von „Verfahrensgerechtigkeit“. Eine Kritik der Procedural-Justice Forschung. Diplomarbeit im Fach Soziologie an der Technischen Hochschule Berlin.

Esser, Josef (1970): Vorverständnis und Methodenwahl in der Rechtsfindung. Rationalitätsgarantien der richterlichen Entscheidungspraxis. Frankfurt a.M.: Athenäum.

Folger, Robert/Cropanzano, Russell (1998): Organizational Justice and Human Resource Management. Thousand Oaks/Kalifornien: Sage.

Greenberg, Jerald/Folger, Robert (1983): Procedural Justice, Participation and the Fair Process Effect in Groups and Organizations, in: Paul B. Paulus (Hrsg.), Basic Group Processes. New York: Springer, 235-256.

Grootelar, Hilke A. M./van den Bos, Kees (2018): How Litigants in Dutch Courtrooms Come to Trust Judges: The Role of Perceived Procedural Justice, Outcome Favorability, and Other Sociolegal Moderators. Law and Society Review 52, 234-268.

Grunwald, Henning (2006): Der Gerichtssaal als „revolutionäre Tribüne“. Ideologische SelbstInszenierung im Medium politischer Prozesse der Weimarer Republik. Paragrana 15, 211-225.

Habermas, Jürgen (1971): Theorie der Gesellschaft oder Sozialtechnologie? Eine Auseinandersetzung mit Niklas Luhmann, in: Jürgen Habermas/Niklas Luhmann, Theorie der Gesellschaft oder Sozialtechnologie - Was leistet die Systemforschung? Frankfurt a.M.: Suhrkamp, 142-290.

Habermas, Jürgen (1976): Legitimationsprobleme im modernen Staat, in: Peter Graf Kielmannsegg (Hrsg.), Legitimationsprobleme politischer Systeme. Opladen: Westdeutscher Verlag, 39-61.

Hagen, Johann J. (1972): Legitimation durch Verfahren. Juristische Schulung 11, 485-488.

Haller, Volkmar/Machura, Stefan (1995): Procedural Justice at German Courts as Seen by Defendants and Juvenile Prisoners. Social Justice Research 8, 197-215.

Heck, Justus (2017): Vermittlung im Zivilverfahren. Zu Funktionen und Folgen undifferenzierter Konfliktbearbeitung, in diesem Heft.

Hennis, Wilhelm (1976): Legitimität, in: Peter Graf Kielmannsegg (Hrsg.), Legitimationsprobleme politischer Systeme. Opladen: Westdeutscher Verlag, 9-38.

Hough, Mike (2013): Procedural Justice and Professional Policing in Times of Austerity. Criminology and Criminal Justice 13, 181-197.

Huq, Aziz Z./Tyler, Tom R./Schulhofer, Stephen J. (2011): Mechanisms for Eliciting Cooperation in Counterterrorism Policing: Evidence from the United Kingdom. Journal of Empirical Legal Studies 8, 728-761.

Jackson, Jonathan/Sunshine, Jason (2007): Public Confidence in Policing. A Neo-Durkheimian Perspective. British Journal of Criminology 47, 214-233.

Jost, Fritz (2017): Strategien der Bewältigung des Wahrheitsproblems im (Zivil-)Prozess, in diesem Heft.

Kieserling, André (2010): Simmels Formen in Luhmanns Verfahren, in: Barbara Stollberg-Rilinger/ André Krischer (Hrsg.), Herstellung und Darstellung von Entscheidungen. Verfahren, Verwalten und Verhandeln in der Vormoderne. Berlin: Duncker \& Humblot, 109-125.

Kieserling, André (2012): Legitimation durch Verfahren (1969), in: Oliver Jahraus/Armin Nassehi/Mario Grizelj et al. (Hrsg.), Luhmann Handbuch. Leben-Werk - Wirkung. Stuttgart: J. B. Metzler, 145-149.

Klenner, Hermann (1976): Die Macht der Ohnmacht. Zur Systemstrukturellen Rechtstheorie. Demokratie und Recht 1, 14-23. 
Köcher, Renate (2014): Großes Vertrauen in die deutsche Justiz. Frankfurter Allgemeinen Zeitung, No. 192, 20. August 2014.

Landis, Jean M./Goodstein, Lynne (1986): When is Justice Fair? An Integrated Approach to the Outcome Versus Procedure Debate. American Bar Foundation Research Journal, 675-707.

Lang, Monika (1993/94): Thesenpapier zum Referat „Niklas Luhmann ,Legitimation durch Verfahren““. Rechtssoziologische Übung „Der Strafprozeß aus Sicht des Angeklagten“. Ruhr-Universität Bochum: Juristische Fakultät, Wintersemester 1993/94.

Lautmann, Rüdiger (1972): Justiz - die stille Gewalt. Frankfurt a.M.: Athenäum Fischer. Leventhal, Gerhard S. (1980): What Should Be Done With Equity Theory?, in: Kenneth J. Gergen/ Martin S. Greenberg/Richard H. Willis (Hrsg.), Social Exchange: Advances in Theory and Research, Vol. 9. New York: Plenum, 27-55.

Liebler, Carina/Zifonun, Dariuš (2017): Interaktionsordnung des Gerichtsverfahrens. Strukturelemente ihres Vollzugs und die Bewältigung von Identitätsproblemen, in diesem Heft.

Lind, E. Allan (1994 a). Justice and Authority Relations in Organizations. American Bar Foundation Working Paper Series, Nr. 9420.

Lind, E. Allan (1994 b): Procedural Justice and Culture: Evidence for Ubiquitous Process Concerns. Zeitschrift für Rechtssoziologie 15, 24-36.

Lind, E. Allan (1994 c): Procedural Justice, Disputing, and Reactions to Legal Authorities. American Bar Foundation Working Paper Series, Nr. 9403.

Lind, E. Allan/Kulik, Carol T./Ambrose, Maureen/Vera Park, Maria V. de (1993): Individual and Corporate Dispute Resolution: Using Procedural Fairness as a Decision Heuristic. Administrative Science Quarterly 38, 224-251.

Lind, E. Allan/Tyler, Tom R. (1988): The Social Psychology of Procedural Justice. New York: Plenum.

Luhmann, Niklas (1981): Selbstlegitimation des Staates, in: Norbert Achenberg/Werner Krawietz (Hrsg.), Legitimation des modernen Staates. Wiesbaden: Steiner, 65-83. Luhmann, Niklas (1983 a): Legitimation durch Verfahren. Frankfurt a.M.: Suhrkamp. Luhmann, Niklas (1983 b): Rechtssoziologie. 2. Auflage. Opladen: Westdeutscher Verlag. Luhmann, Niklas (1985): A Sociological Theory of Law, edited by Martin Albrow. London Routledge and Kegan Paul.

Luhmann, Niklas (1995 a): Das Recht der Gesellschaft. Frankfurt a.M.: Suhrkamp.

Luhmann, Niklas (1995 b): Was ist Kommunikation?, in: ders., Soziologische Aufklärung 6. Die Soziologie und der Mensch. Opladen: Westdeutscher Verlag, 113-124.

Luhmann, Niklas (1999 a): Gerechtigkeit in den Rechtssystemen der modernen Gesellschaft, in: ders., Ausdifferenzierung des Rechts. Frankfurt a.M.: Suhrkamp, 374-418.

Luhmann, Niklas (1999 b). Positivität des Rechts als Voraussetzung einer modernen Gesellschaft, in: ders., Ausdifferenzierung des Rechts. Frankfurt a.M.: Suhrkamp, 113-153. Luhmann, Niklas (1999 c): Recht als soziales System. Zeitschrift für Rechtssoziologie 20, 1-13. Luhmann, Niklas/Hagen, Wolfgang (2005): Es gibt keine Biografie. Niklas Luhmann im Radiogespräch mit Wolfgang Hagen, in: Wolfgang Hagen (Hrsg.), Warum haben Sie keinen Fernseher, Herr Luhmann? Letzte Gespräche mit Niklas Luhmann. Berlin: Kadmos, 13-48. Machura, Stefan (1993): Niklas Luhmanns „Legitimation durch Verfahren“ im Spiegel der Kritik. Zeitschrift für Rechtssoziologie 14, 97-114.

Machura, Stefan (2001): Fairneß und Legitimität. Baden-Baden: Nomos.

Machura, Stefan (2005): Procedural Unfairness in Real and Film Trials: Why Do Audiences Understand Stories Placed in Foreign Legal Systems?, in: Michael Freeman (Hrsg.), Law and Popular Culture. Oxford: Oxford University Press, 148-159. 
Machura, Stefan (2006): Ehrenamtliche Verwaltungsrichter. Berlin: Lit.

Machura, Stefan (2013): Ministerialbürokratie, in: Uwe Andersen/Wichard Woyke (Hrsg.), Handwörterbuch des politischen Systems der Bundesrepublik Deutschland. 7. Auflage Wiesbaden: VS, 453-456.

Machura, Stefan (2016): Inter- and Intra-agency Cooperation in Safeguarding Children. A Staff Survey. British Journal of Social Work 46, 652-668.

Machura, Stefan/Donskow, Dmitrij/Litvinova, Olga (2003): Ehrenamtliche Richter in Südrussland. Münster: Lit.

Machura, Stefan/Love, Thomas/Dwight, Adam (2014): Law Students' Trust in the Courts and the Police. International Journal of Law, Crime and Justice, 42, 287-305.

Offe, Claus (1972): Strukturprobleme des kapitalistischen Staates. Frankfurt a.M.: Suhrkamp. Platow, Michael J./Reid, Scott/Andrew, Sarah (1998): Leadership Endorsement: The Role of Distributive and Procedural Behavior in Interpersonal and Intergroup Contexts. Group Processes and Intergroup Relations 1, 35-47.

Puttfarken, Hans-Jürgen (1977): Gegenwartsprobleme der deutschen Zivilrechtswissenschaft. Juristische Schulung 8, 493-499.

Rasinski, Kenneth A./Tyler, Tom R. (1988): Fairness and Vote Choice in the 1984 Presidential Election. American Politics Quarterly 16, 5-24.

Röhl, Klaus F. (1977): Gegenwartsströmungen der Rechtssoziologie. Informationsbrief Rechtssoziologie, hrsg. von der Sektion Rechtssoziologie in der Deutschen Gesellschaft für Soziologie und dem Arbeitskreis für Rechtssoziologie e.V., Sonderheft 1. Berlin: Eigenverlag.

Röhl, Klaus F. (1993): Verfahrensgerechtigkeit (Procedural Justice). Einführung in den Themenbereich und Überblick. Zeitschrift für Rechtssoziologie 14: 1-34.

Röhl, Klaus F. (1994): Procedural Justice: Zur Sozialpsychologie des Verfahrens, in: Hagen Hof et al. (Hrsg.), Recht und Verhalten. Baden-Baden, 57-70.

Röhl, Klaus F./Weiß, Matthias (2005): Die obligatorische Streitschlichtung in der Praxis. Münster: Lit.

Schaper, Jürgen (1985): Studien zur Theorie und Soziologie des gerichtlichen Verfahrens. Ein Beitrag zur Diskussion um Grundlagen und Grundbegriffe von Prozeß und Prozeßrecht. Berlin: Duncker \& Humblot.

Sunshine, Jason/Tyler, Tom R. (2003): The Role of Procedural Justice and Legitimacy in Shaping Public Support for Policing. Law and Society Review 37, 513-547.

Starystach, Sebastian (2017): Zur Funktion informeller Kommunikation in Gerichtverfahren am Beispiel des deutschen Strafverfahrens, in diesem Heft.

Tausch, Anne-Marie/Langer, Inghard (1971): Soziales Verhalten von Richtern gegenüber Angeklagten; Merkmale, Auswirkungen sowie Änderungen durch ein Selbst-Training. Zeitschrift für Entwicklungspsychologie und Pädagogische Psychologie 3, 283-303.

Tausch, Anne-Marie/Schiefelbein-Heitmann, Mira/Schwab, Reinhold/Doßmann, Angelika (1975): Psychische Änderungen und Gesprächsverhalten in personenzentrierten Encountergruppen mit Gefängnisinsassen, Richtern, Psychotherapieklienten und Psychologen. Psychologie in Erziehung und Unterricht 22, 161-171.

Thibaut, John/Walker, Laurens (1975): Procedural Justice. Hillsdale/N.J: Erlbaum.

Treibel, Annette (1993): Einführung in soziologische Theorien der Gegenwart. Opladen: Leske und Budrich.

Tyler, Tom R. (1984): The Role of Perceived Injustice in Defendants> Evaluations of Their Courtroom Experience. Law and Society Review 18, 51-74. 
Tyler, Tom R. (1990): Why People Obey the Law. New Haven: Yale University Press.

Tyler, Tom R. (1994). The Psychology of Legitimacy, American Bar Foundation Working Paper Series, Nr. 9425.

Tyler, Tom R./Belliveau, Maura A. (1995): Tradeoffs in Justice Principles: Definitions of Fairness, in: Barbara Benedict Bunker/Jeffrey Z. Rubin (Hrsg.), Conflict, Cooperation, and Justice. San Francisco: Jossey-Bass, 291-314.

Tyler, Tom R./Huo, Yuen J. (2002): Trust in the Law. Encouraging Public Cooperation with the Police and Courts. Russell Sage Foundation: New York.

Tyler, Tom R./Jackson, Jonathan (2013): Future Challenges in the Study of Legitimacy and Criminal Justice, in: Justice Tankebe/Alison Liebling (Hrsg.), Legitimacy and Criminal Justice: an International Exploration. Oxford: Oxford University Press, 105-126.

Tyler, Tom R./Lind, E. Allan (1992): A Relational Model of Authority in Groups, in: Mark Zanna (Hrsg.), Advances in Experimental Social Psychology, Vol. 25. New York: Academic, 115-191.

Vermunt, Riel/Blaauw, Eric/Lind, Allan (1996): Fairness Evaluations of Encounters with Police Officers and Correctional Officers. American Bar Foundation Working Paper Series, Nr. 9602. Voigt, Rüdiger (1998): Thesen zum Thema „Rechtspolitische Kommunikation“, in: Johannes W. Pichler (Hrsg.), Rechtsakzeptanz und Handlungsorientierung. Wien: Böhlau, 125-132. Walster, Elaine H./Berscheid, Ellen/Walster, G. William (1973): New Directions in Equity Research. Journal of Personality and Social Psychology 25, 151-176

Weber, Max (1980): Wirtschaft und Gesellschaft, hrsg. von Johannes Winckelmann. Tübingen: Mohr.

Welsh, Lucy (2017): The Effects of Changes to Legal Aid on Lawyers' Professional Identity and Behaviour in Summary Criminal Cases: A Case Study. Journal of Law and Society 44, 559-585.

Zippelius, Reinhold (1973): Legitimation durch Verfahren?, in: Gotthard Paulus/ Uwe Diederichsen/Claus-Wilhelm Canaris (Hrsg.), Festschrift für Karl Larenz zum 70. Geburtstag. München: Beck, 293-304.

\section{Autoreninformationen:}

\section{Professor Dr. Dr. habil. Stefan Machura}

Bangor University, Eollege of Arts, Humanities and Business, School of History, Philosophy and Social Sciences, Bangor, Gwynedd, LL57 2DG, email: s.machura@bangor.ac.uk

Stefan Machura, Dr. rer. soc., Dr. rer. pol. habil., ist Professor of Criminology and Criminal Justice an der Bangor University. Er hat an der Ruhr-Universität Bochum Soziologie und Politikwissenschaft studiert und nach der Promotion schwerpunktmäßig Rechtssoziologie und Politische Soziologie unterrichtet. Mit Klaus F. Röhl hat er den Band „Procedural Justice“ (Aldershot: Ashgate 1997) herausgegeben. Weitere Veröffentlichungen sind u. a. „Politik und Verwaltung" (hrsg. mit Knut Papendorf und Kristian Andenæs), Opladen: VS 2005; „Understanding Law in Society ;s. mit Knut Papendorfund Anne Hellum), Zürich: Lit. 2011; „Eugen Ehrlich's Sociolosy U. Law” - -.. ich: Lit 2014; „ „Oxford Encyclopedia on Crime, Media, and Popular Culture" (hrsg. mit N Rafter/Michelle Brown et al.), New York: Oxford University Press 2018. 